\title{
ON THE INTRINSIC DESZCZ SYMMETRIES AND THE EXTRINSIC CHEN CHARACTER OF WINTGEN IDEAL SUBMANIFOLDS
}

\author{
S. DECU, M. PETROVIĆ-TORGAŠEV, A. ŠEBEKOVIĆ AND L. VERSTRAELEN
}

\begin{abstract}
In this paper it is shown that all Wintgen ideal submanifolds in ambient real space forms are Chen submanifolds. It is also shown that the Wintgen ideal submanifolds of dimension $>3$ in real space forms do intrinsically enjoy some curvature symmetries in the sense of Deszcz of their Riemann-Christoffel curvature tensor, of their Ricci curvature tensor and of their Weyl conformal curvature tensor.
\end{abstract}

\section{Wintgen ideal submanifolds}

Let $M^{n}$ be an $n$-dimensional Riemannian submanifold of an $(n+m)$-dimensional real space form $\tilde{M}^{n+m}(c)$ of curvature $c,(n \geq 2, m \geq 1)$. Let $g$ and $\nabla$, and, respectively, $\tilde{g}$ and $\tilde{\nabla}$, denote the Riemannian metrics and the corresponding Levi-Civita connections of $M^{n}$ and of $\tilde{M}^{n+m}(c)$. The formulae of Gauss and Weingarten are then given by

$$
\tilde{\nabla}_{X} Y=\nabla_{X} Y+h(X, Y)
$$

and

$$
\tilde{\nabla}_{X} \xi=-A_{\xi} X+\nabla_{X}^{\perp} \xi
$$

whereby $h, A_{\xi}$ and $\nabla^{\perp}$ denote the second fundamental form, the shape operator or Weingarten map with respect to $\xi$ and the normal connection of $M^{n}$ in $\tilde{M}^{n+m}(c)$, respectively, $\left(X, Y\right.$, etc. stand for tangent vector fields and $\xi$ etc. for normal vector fields on $M^{n}$ in $\left.\tilde{M}^{n+m}(c)\right)$. From (1) and (2) it follows that

$$
\tilde{g}(h(X, Y), \xi)=g\left(A_{\xi}(X), Y\right),
$$

Corresponding author: M. Petrović-Torgašev.

Received April 12, 2009.

2000 Mathematics Subject Classification. Primary 53B25, Secondary 53C42.

Key words and phrases. Submanifolds, Wintgen ideal submanifolds, Chen submanifolds, Deszcz symmetric manifolds.

S. Decu and A. Šebeković were partially supported by the Simon Stevin Institute for Geometry. L. Verstraelen was partially supported by the Research Foundation - Flanders project G.0432.07 
such that, for any orthonormal local normal frame $\left\{\xi_{\alpha}\right\}$ on $M^{n}$ in $\tilde{M}^{n+m}(c),(\alpha, \beta, \ldots \in$ $\{1,2, \ldots, m\})$ :

$$
h(X, Y)=\sum_{\alpha} g\left(A_{\alpha}(X), Y\right) \xi_{\alpha}
$$

whereby $A_{\alpha}=A_{\xi_{\alpha}}$. The mean curvature vector field $\vec{H}$ of $M^{n}$ in $\tilde{M}^{n+m}(c)$ is defined as $\vec{H}=\frac{1}{n} \operatorname{tr} h=\frac{1}{n} \sum_{i=1}^{n} h\left(E_{i}, E_{i}\right)$, for any orthonormal local tangent frame $\left\{E_{i}\right\}$ on $M^{n},(i, j, \ldots \in\{1,2, \ldots, n\})$, and its length $H=\|\vec{H}\|$ is the mean curvature of $M^{n}$ in $\tilde{M}^{n+m}(c)$.

Let $R$ denote the $(0,4)$ Riemann-Christoffel curvature tensor of $\left(M^{n}, g\right)$. Then, according to the equation of Gauss,

$$
\begin{aligned}
R(X, Y, Z, W)= & \tilde{g}(h(Y, Z), h(X, W))-\tilde{g}(h(X, Z), h(Y, W)) \\
& +c\{g(Y, Z) g(X, W)-g(X, Z) g(Y, W)\} .
\end{aligned}
$$

Denoting by $\tau$ the scalar curvature function of $\left(M^{n}, g\right)$, we have

$$
\tau(p):=\sum_{i<j} K\left(p, E_{i}(p) \wedge E_{j}(p)\right)
$$

whereby $K\left(p, E_{i}(p) \wedge E_{j}(p)\right)$ denotes the sectional curvature of $\left(M^{n}, g\right)$ at a point $p$ of $M^{n}$ for the plane section $\pi=E_{i}(p) \wedge E_{j}(p)$ in $T_{p} M^{n}$. By $K_{\text {inf }}$ we will further denote the function $K_{\text {inf }}: M \rightarrow R: p \mapsto K_{\text {inf }}(p):=$ the minimal value of all sectional curvatures of $M$ at $p$.

The normalised scalar curvature $\rho$ of the Riemannian manifold $M^{n}$ is defined to be

$$
\rho=\frac{2}{n(n-1)} \sum_{i<j} R\left(E_{i}, E_{j}, E_{j}, E_{i}\right) .
$$

By the equation of Ricci, the normal curvature tensor $R^{\perp}$ of $M$ in $\tilde{M}$ is given as follows:

$$
R^{\perp}(X, Y ; \xi, \eta):=\tilde{g}\left(R^{\perp}(X, Y) \xi, \eta\right)=g\left(\left[A_{\xi}, A_{\eta}\right] X, Y\right),
$$

whereby $R^{\perp}(X, Y):=\nabla_{X}^{\perp} \nabla_{Y}^{\perp}-\nabla_{Y}^{\perp} \nabla_{X}^{\perp}-\nabla_{[X, Y]}^{\perp}$ and $\left[A_{\xi}, A_{\eta}\right]:=A_{\xi} A_{\eta}-A_{\eta} A_{\xi}$.

The normalised scalar normal curvature $\rho^{\perp}$ of $M$ in $\tilde{M}$ is then defined to be

$$
\rho^{\perp}=\frac{2}{n(n-1)}\left\{\sum_{i<j} \sum_{\alpha<\beta}\left[R^{\perp}\left(E_{i}, E_{j} ; \xi_{\alpha}, \xi_{\beta}\right)\right]^{2}\right\}^{\frac{1}{2}} .
$$

We remark that $\rho^{\perp}=0$ if and only if the normal connection is flat, which, as follows from (8) and as already observed by Cartan [2], is equivalent to the simultaneous diagonalisability of all shape operators $A_{\xi}$ of $M^{n}$ in $\tilde{M}^{n+m}$.

For surfaces $M^{2}$ in $E^{3}$, the Euler inequality $K \leq H^{2}$, whereby $K$ is the intrinsic Gauss curvature of $M^{2}$ and $H^{2}$ is the extrinsic squared mean curvature of $M^{2}$ in $E^{3}$, at once follows from the fact that $K=k_{1} k_{2}$ and $H=\frac{1}{2}\left(k_{1}+k_{2}\right)$ whereby $k_{1}$ and $k_{2}$ denote 
the principal curvatures of $M^{2}$ in $E^{3}$. And, obviously, $K=H^{2}$ everywhere on $M^{2}$ if and only if the surface $M^{2}$ is totally umbilical in $E^{3}$, i.e. $k_{1}=k_{2}$ at all points of $M^{2}$, or still, by a theorem of Meusnier, if and only if $M^{2}$ is a part of a plane $E^{2}$ or of a round sphere $S^{2}$ in $E^{3}$. In the late 19 seventies, Wintgen proved that the Gauss curvature $K$ and the squared mean curvature $H^{2}$ and the normal curvature $K^{\perp}$ of any surface $M^{2}$ in $E^{4}$ always satisfy the inequality

$$
K \leq H^{2}-K^{\perp},
$$

and that actually the equality holds if and only if the curvature ellipse of $M^{2}$ in $E^{4}$ is a circle [24]. We recall that the ellipse of curvature at a point $p$ of $M$ is defined as $\mathcal{E}_{p}=\left\{h(X, X) \mid X \in T_{p} M,\|X\|=1\right\}$. This Wintgen inequality between the most important intrinsic and extrinsic scalar valued curvatures of surfaces $M^{2}$ in $E^{4}$ was shown to hold more generally for all surfaces $M^{2}$ in arbitrary dimensional space forms $\tilde{M}^{2+m}(c)$, inclusive the above characterisation of the equality case, by Rouxel in 1981 [18] and by Guadalupe and Rodriguez in 1983 [11]. After these extensions of Wintgen inequality (10), in 1999 De Smet, Dillen, Vrancken and one of the authors [6] proved the Wintgen inequality $\rho \leq H^{2}-\rho^{\perp}+c$ for all submanifolds $M^{n}$ of codimension 2 in all real space forms $\tilde{M}^{n+2}(c)$ and characterised the equality as follows in terms of the shape operators.

Theorem A. For any submanifold $M^{n}$ of arbitrary dimension $n$ and codimension 2 in a real space form $\tilde{M}^{n+2}(c)$ of curvature $c$, at every point $p$ of $M^{n}$ :

$$
\rho \leq H^{2}-\rho^{\perp}+c,
$$

and equality holds if and only if there exist orthonormal bases of the tangent space $T_{p} M$ and the normal space $T_{p}^{\perp} M$ with respect to which the corresponding Weingarten maps are given by

$$
A_{1}=\left(\begin{array}{ccccc}
\lambda & \mu & 0 & \ldots & 0 \\
\mu & \lambda & 0 & \ldots & 0 \\
0 & 0 & \lambda & \ldots & 0 \\
\vdots & \vdots & \vdots & & \vdots \\
0 & 0 & 0 & \ldots & \lambda
\end{array}\right), \quad A_{2}=\left(\begin{array}{ccccc}
\mu & 0 & 0 & \ldots & 0 \\
0 & -\mu & 0 & \ldots & 0 \\
0 & 0 & 0 & \ldots & 0 \\
\vdots & \vdots & \vdots & & \vdots \\
0 & 0 & 0 & \ldots & 0
\end{array}\right),
$$

for some $\lambda, \mu \in R$.

We remark that, in the case of trivial normal connection, (11) reduces to Chen's inequality $\rho \leq H^{2}+c$ established in [3]. The Wintgen inequality (11) was conjectured to hold for all submanifolds $M^{n}$ in all real space forms $\tilde{M}^{n+m}(c)$ in the same paper [6], and this is called "the DDVV conjecture" or "the conjecture on Wintgen's inequality". Recently, Choi and $\mathrm{Lu}[5]$ proved that this conjecture is true for all 3-dimensional submanifolds of arbitrary dimensional real space forms $\tilde{M}^{3+m}(c),(m \geq 2)$, and very recently, and 
independently, Lu [16] and Ge and Tang [10], settled this conjecture in general. From [10] we recall the final result in this respect.

Theorem B. For any submanifold $M^{n}$ of arbitrary dimension $n, n \geq 2$, and with arbitrary codimension $m, m \geq 2$ in a real space form $\tilde{M}^{n+m}(c)$ of curvature $c$, at every point $p$ of $M^{n}$ :

$$
\rho \leq H^{2}-\rho^{\perp}+c,
$$

and equality holds if and only if there exist orthonormal bases of the tangent space $T_{p} M$ and the normal space $T_{p}^{\perp} M$ with respect to which the corresponding Weingarten maps are given by

$$
A_{1}=\left(\begin{array}{ccccc}
\lambda & \mu & 0 & \ldots & 0 \\
\mu & \lambda & 0 & \ldots & 0 \\
0 & 0 & \lambda & \ldots & 0 \\
\vdots & \vdots & \vdots & & \vdots \\
0 & 0 & 0 & \ldots & \lambda
\end{array}\right), \quad A_{2}=\left(\begin{array}{ccccc}
\mu & 0 & 0 & \ldots & 0 \\
0 & -\mu & 0 & \ldots & 0 \\
0 & 0 & 0 & \ldots & 0 \\
\vdots & \vdots & \vdots & & \vdots \\
0 & 0 & 0 & \ldots & 0
\end{array}\right)
$$

for some $\lambda, \mu \in R$, and all other shape operators do vanish identically.

Submanifolds satisfying the equality in the Wintgen inequality (12) are called Wintgen ideal submanifolds. A justification for the terminology "Wintgen ideal submanifolds" $M^{n}$ in $\tilde{M}^{n+m}(c)$ for those submanifolds $M^{n}$ in $\tilde{M}^{n+m}(c)$ for which $\rho=H^{2}-\rho^{\perp}+c$ holds at all points $p$ of $M^{n}$, is as follows: for all possible isometric immersions of $M^{n}$ in space forms $\tilde{M}^{n+m}(c)$, the value of the intrinsic scalar curvature $\rho$ of $M$ puts a lower bound to all possible values of the extrinsic curvature $H^{2}-\rho^{\perp}+c$ that $M$ in any case can not avoid to "undergo" as a submanifold in $\tilde{M}$. And, from this point of view, $M$ is called a Wintgen ideal submanifold, when it actually is able to achieve a realisation in $\tilde{M}$ such that this extrinsic curvature indeed everywhere assumes its theoretically smallest possible value as given by its intrinsic normalised scalar curvature.

\section{Deszcz symmetries of Wintgen ideal submanifolds}

For a Riemannian manifold $\left(M^{n}, g\right)$, let $R$ also denote the $(1,1)$ curvature operator $R(X, Y):=\nabla_{X} \nabla_{Y}-\nabla_{Y} \nabla_{X}-\nabla_{[X, Y]}$, besides the $(0,4)$ curvature tensor, such that, by definition

$$
R(X, Y, Z, W)=g(R(X, Y) Z, W)
$$

By the action of the curvature operator $R$ working as a derivation on the curvature tensor $R$, the following $(0,6)$ tensor $R \cdot R$ is obtained:

$$
\begin{array}{r}
(R \cdot R)\left(X_{1}, X_{2}, X_{3}, X_{4} ; X, Y\right):=(R(X, Y) \cdot R)\left(X_{1}, X_{2}, X_{3}, X_{4}\right) \\
=-R\left(R(X, Y) X_{1}, X_{2}, X_{3}, X_{4}\right)-R\left(X_{1}, R(X, Y) X_{2}, X_{3}, X_{4}\right) \\
-R\left(X_{1}, X_{2}, R(X, Y) X_{3}, X_{4}\right)-R\left(X_{1}, X_{2}, X_{3}, R(X, Y) X_{4}\right) .
\end{array}
$$


It was recently shown by one of the authors and Haesen [12], that this tensor $R \cdot R$ can be geometrically interpreted as giving the second order measure of the change of the sectional curvatures $K(p, \pi)$ for tangent $2 D$-planes $\pi$ at points $p$ after the parallel transport of $\pi$ all around infinitesimal co-ordinate parallelograms in $M$ cornered at $p$. Thus, according to [12], the semi-symmetric or Szabó symmetric spaces ([20] [21]), i.e. the spaces satisfying $R \cdot R=0$, are the Riemannian manifolds $\left(M^{n}, g\right)$ for which all sectional curvatures remain preserved after parallel transport of their planes around all infinitesimal co-ordinate parallelograms in $M$. The locally symmetric or Cartan symmetric spaces, i.e. the Riemannian manifolds $\left(M^{n}, g\right)$ for which $\nabla R=0$, constitute a proper subclass of the Szabó symmetric spaces. Deszcz symmetric spaces or pseudo-symmetric spaces ([7] [23]) are characterised by the fact that their $(0,6)$ curvature tensor $R \cdot R$ is proportional to their $(0,6)$ Tachibana tensor $Q(g, R):=-\wedge_{g} \cdot R$, whereby the metrical endomorphism $\wedge_{g}$ acts on the $(0,4)$ tensor $R$ as a derivation, i.e. by the fact that

$$
R \cdot R=L Q(g, R),
$$

for some function $L: M^{n} \rightarrow R$, (whenever $Q(g, R) \neq 0$ ). We recall that $Q(g, R) \equiv 0$ characterises the real space forms.

From [12] we further mention the following. Two 2-planes $\pi$ and $\bar{\pi}$, spanned by vectors $\vec{u}, \vec{v}$ and $\vec{x}, \vec{y}$ respectively, at a same point $p$ of $M$, are said to be curvature dependent if $Q(g, R)(\vec{u}, \vec{v}, \vec{v}, \vec{u} ; \vec{x}, \vec{y}) \neq 0$, which condition is independent of the choices of bases for $\pi$ and $\bar{\pi}$. For such planes, the double sectional curvature or the sectional curvature of Deszcz or the Riemann curvature of Deszcz $L(p, \pi, \bar{\pi})$ is defined as the real number given by

$$
L(p, \pi, \bar{\pi}):=\frac{(R \cdot R)(\vec{u}, \vec{v}, \vec{v}, \vec{u} ; \vec{x}, \vec{y})}{Q(g, R)(\vec{u}, \vec{v}, \vec{v}, \vec{u} ; \vec{x}, \vec{y})},
$$

(which is independent of the choices of bases for $\pi$ and $\bar{\pi}$ ); it is a scalar valued Riemannian invariant. The knowledge of the tensor $R \cdot R$ is equivalent to the knowledge of the sectional curvatures $L(p, \pi, \bar{\pi})$ of Deszcz. And just like the geometrical interpretation of the sectional curvatures $K(p, \pi)$ of Riemann in terms of the parallelogramoïds of Levi-Civita [15], also the sectional curvatures $L(p, \pi, \bar{\pi})$ of Deszcz can be interpreted in these terms (in this respect, we refer to [13] where in particular such interpretations are obtained for the sectional curvatures as well as for the Ricci and conformal Weyl curvatures of Deszcz in terms of the squaroïds of Levi-Civita). Finally the Deszcz symmetric spaces are characterised by the isotropy of the curvatures $L(p, \pi, \bar{\pi})$, i.e. by the property that at every point $p$ of $M$ the scalars $L(p, \pi, \bar{\pi})$ are the same for all possible pairs of curvature dependent tangent planes $\pi$ and $\bar{\pi}$ at $p$. In the present situation however there is no lemma of Schur, which then would further force this real valued function $L: M \rightarrow R$ automatically to be constant; therefore, Kowalski and Sekizawa called the pseudo-symmetric spaces for which the double sectional curvature $L$ is indeed a constant, independent of the planes $\pi$ and $\bar{\pi}$ as well as of the points $p$ of $M$, the pseudo-symmetric spaces of constant type $L$ [14]. For instance, the standard models of the Thurston geometries [22] are the $3 D$-prototypes of the Deszcz-symmetric spaces with constant $L$ [1]. 
And similar studies concerning, in particular, the $(0,4)$ Weyl conformal curvature tensor $C$ and the $(0,2)$ Ricci tensor $S$ have been carried through in the mean time, (characterising the corresponding "Deszcz-symmetries" in terms of the isotropy of the corresponding scalar curvature functions which depend on two planes and on a plane and a direction, respectively). For a recent general exposition on conditions of Deszcz symmetry we refer to $[8]$.

It was shown in [17] that Wintgen ideal submanifolds $M^{n}$ of dimension $n>3$ and with codimension 2 in real space forms $\tilde{M}^{n+2}(c)$ of curvature $c$ intrinsically enjoy some curvature symmetries in the sense of Deszcz, i.e. the Deszcz symmetries of their RiemannChristoffel curvature tensor $R$, of their Ricci curvature tensor $S$ and of their conformal curvature tensor of Weyl C. Such Wintgen ideal submanifolds $M^{n}$ of $\tilde{M}^{n+2}(c)$ are Deszcz symmetric if and only if $M^{n}$ is totally umbilical in $\tilde{M}^{n+2}(c)$, in which case $L=0$, or $M^{n}$ is minimal in $\tilde{M}^{n+2}(c)$, in which case $L=c$. Moreover, it was proved in [9] that the Deszcz symmetry, or, equivalently, the property to be quasi-Einstein, for 3D-Wintgen ideal submanifolds $M^{3}$ in $\tilde{M}^{3+m}(c)$, can be characterised in terms of the intrinsic minimal values of the Ricci curvatures of $M$ and of the extrinsic notions of the umbilicity, the minimality and the pseudo-umbilicity of such $M^{3}$ in $\tilde{M}^{3+m}(c)$. Therefore, concerning the study of Deszcz symmetries of Wintgen ideal submanifolds only the situation of dimension $n>3$ in case of arbitrary codimension $m$ remains to be considered. But, in view of Theorems A and B, the proofs given in [17] obviously also hold for the general codimensions, so that accordingly we can announce the following general results.

Theorem 1. A Wintgen ideal submanifold $M^{n}$ of a real space form $\tilde{M}^{n+m}(c),(n>$ $3, m \geq 2)$ is Deszcz symmetric, if and only if $M^{n}$ is totally umbilical in $\tilde{M}^{n+m}(c)$, in which case $L=0$, or $M^{n}$ is minimal in $\tilde{M}^{n+m}(c)$, in which case $L=c$.

Theorem 2. A Wintgen ideal submanifold $M^{n}$ of $\tilde{M}^{n+m}(c),(n>3, m \geq 2)$, is Deszcz Ricci-symmetric, i.e. satisfies $R \cdot S=L_{S} Q(g, S)$ for some function $L_{S}: M^{n} \rightarrow R$, if and only if $M^{n}$ is Deszcz symmetric.

Theorem 3. Every Wintgen ideal submanifold $M^{n}$ of $\tilde{M}^{n+m}(c),(n>3, m \geq 2)$, is a Riemannian manifold with pseudo-symmetric conformal Weyl tensor, i.e. satisfies $C \cdot C=L_{C} Q(g, C)$ for some function $L_{C}: M^{n} \rightarrow R$.

Proposition 4. A Wintgen ideal submanifold $M^{n}$ of $\tilde{M}^{n+m}(c),(n>3, m \geq 2)$ is minimal if and only if the pseudo-symmetry function of its Weyl conformal curvature tensor is given by

$$
L_{C}=\frac{n-3}{(n-1)(n-2)}\left(c-K_{\text {inf }}\right)
$$

\section{Chen submanifolds}

For submanifolds $M^{n}$ of $\tilde{M}^{n+m}$ the notion of allied vector field of a given normal vector field of $M^{n}$ is defined in [4] and, accordingly, for any submanifold $M^{n}$ in $\tilde{M}^{n+m}$, 
for a local orthonormal frame $\left\{\xi_{1}=\frac{\vec{H}}{\|\vec{H}\|}, \xi_{2}, \ldots, \xi_{m}\right\}$ whereby $\vec{H}$ is the mean curvature vector field of $M^{n}$ in $\tilde{M}^{n+m}$,

$$
a(\vec{H})=\frac{1}{n} \sum_{\beta=2}^{m} \operatorname{tr}\left(A_{1} A_{\beta}\right) \xi_{\beta},
$$

is the allied vector field of $\vec{H}$ or allied mean curvature vector field of $M^{n}$ in $\tilde{M}^{n+m}$. A submanifold $M^{n}$ is called an $\mathcal{A}$-submanifold or a Chen submanifold if the allied mean curvature vector field of $M^{n}$ identically vanishes, $a(\vec{H}) \equiv \overrightarrow{0}$. By a result of B. Rouxel [19], a submanifold $M^{n}$ of $\tilde{M}^{n+m}$ is a Chen submanifold if and only if the mean curvature vector at any point $p$ of $M, \vec{H}(p)$, is an axis of symmetry of the $(m-2)$-nd polar of its Kommerell hyperquadric curvature image in the normal space $T_{p}^{\perp} M$. Minimal submanifolds, pseudo-umbilical submanifolds and hypersurfaces are Chen submanifolds in a trivial way.

For Wintgen ideal submanifolds $M^{n}$ in real space forms $\tilde{M}^{n+m}(c)$, from the specific forms of the shape operators of these submanifolds given in Theorem B, we have

$$
A_{1} A_{2}=\left(\begin{array}{ccccc}
\lambda \mu-\mu^{2} & 0 & \ldots & 0 \\
\mu^{2}-\lambda \mu & 0 & \ldots & 0 \\
0 & 0 & 0 & \ldots & 0 \\
\vdots & \vdots & \vdots & & \vdots \\
0 & 0 & 0 & \ldots & 0
\end{array}\right), \quad A_{1} A_{\gamma}=0,(\gamma \in\{3, \ldots, m\}),
$$

such that their allied mean curvature vector field $a(\vec{H})$ clearly always is identically zero. This yields the following.

Theorem 5. Every Wintgen ideal submanifold $M^{n}$ of arbitrary dimension $n \geq 2$ and codimension $m \geq 2$ in a real space form $\tilde{M}^{n+m}(c)$, is a Chen submanifold of $\tilde{M}^{n+m}(c)$.

\section{References}

[1] M. Belkhelfa, R. Deszcz and L. Verstraelen, Symmetry Properties of 3-dimensional D'Atri Spaces, Kyungpook Math. J. 46 (2006), 367-376.

[2] E. Cartan, Leçons sur la géométrie des espaces de Riemann, Gauthier-Villars, Paris, 1928.

[3] B. Y. Chen, Mean curvature and shape operator of isometric immersions in real space forms, Glasgow Math. J. 38 (1996), 87-97.

[4] B. Y. Chen, Geometry of Submanifolds, Marcel Dekker, New York, 1973.

[5] T. Choi and Z. Lu, On the DDVV conjecture and the comass in calibrated geometry (I), Math. Z. 260 (2008), 409-429.

[6] P. J. De Smet, F. Dillen, L. Verstraelen and L. Vrancken, A pointwise inequality in submanifold theory, Arch. Math. (Brno) 35 (1999), 115-128.

[7] R. Deszcz, On pseudosymmetric spaces, Bull. Soc. Math. Belg., Série A, 44 (1992), 1-34. 
[8] R. Deszcz, S. Haesen and L. Verstraelen, On natural symmetries, Chapter 6 in Topics in Differential Geometry, Ed. Rom. Acad. Sci., Bucharest (2008).

[9] R. Deszcz, M. Petrović-Torgašev, Z. Șentürk, L. Verstraelen, Characterisation of the pseudo-symmetries of ideal Wintgen submanifolds of dimension 3, (preprint).

[10] J. Ge and Z. Tang, A proof of the DDVV conjecture and its equality case, Pacific J. Math. 237 (2008), 87-95.

[11] I. V. Guadalupe and L. Rodriguez, Normal curvature of surfaces in space forms, Pacific J. Math. 106 (1983), 95-103.

[12] S. Haesen and L. Verstraelen, Properties of a scalar curvature invariant depending on two planes, manuscripta math. 122 (2007), 59-72.

[13] B. Jahanara, S. Haesen, M. Petrović-Torgašev and L. Verstraelen, On the Weyl conformal curvature of Deszcz, Publ. Math. Debrecen 74 (2009), 417- 431.

[14] O. Kowalski and M. Sekizawa, Pseudo-symmetric spaces of constant type in dimension three-elliptic spaces, Rend. Mat. Appl. (7) 17 (1997), 477-512.

[15] T. Levi-Civita, Nozione di parallelismo in una varietá qualcunque e conseguente spezificazione geometrica della curvatura Riemanniana, Rend. Circ. Matem. Palermo 42 (1917), 173-204.

[16] Z. Lu, On the DDVV conjecture and the comass in calibrated geometry (II), arXiv: 0708.2921 (2007).

[17] M. Petrović-Torgašev and L. Verstraelen, On Deszcz symmetries of Wintgen ideal submanifolds, Arch. Math. (Brno) 44 (2008), 57-67.

[18] B. Rouxel, Sur une famille de A-surfaces d'un espace euclidien $E^{4}$, Proc.10 Österreichischer Mathematiker Kongress, Insbruck, 1981, 185.

[19] B. Rouxel, A-submanifolds in Euclidean space, Kodai Math. J. 4 (1981), 181-188.

[20] Z. Szabó, Structure theorems on Riemannian spaces satisfying $R(X, Y) \cdot R=0$. I. The local version, J. Diff. Geom. 17 (1982), 531-582.

[21] Z. Szabó, Structure theorems on Riemannian spaces satisfying $R(X, Y) \cdot R=0$. II. The global version, Geom. Dedicata 19 (1985), 65-108.

[22] W. M. Thurston, Three-dimensional Geometry and Topology, Vol. I, Princeton University Press, Princeton, 1997.

[23] L. Verstraelen, Comments on the pseudo-symmetry in the sense of Deszcz, in Geometry and Topology of Submanifolds, Vol. VI, (eds. F. Dillen e.a.), World Sci. Publ. Co, Singapore, 1994, 119-209.

[24] P. Wintgen, Sur l'inégalité de Chen-Willmore, C. R. Acad. Sci. Paris 288 (1979), 993-995.

University of Bucharest, Faculty of Mathematics, Str. Academiei 1, 010014 Bucharest, Romania. E-mail: simona.decu@gmail.com

University of Kragujevac, Department of Mathematics, Faculty of Science, Radoja Domanovića 12, 34000 Kragujevac, Serbia.

E-mail: mirapt@kg.ac.rs

University of Kragujevac, Technical Faculty, Svetog Save 65, 32000 Čačak, Serbia.

E-mail: sebek@tfc.kg.ac.rs

Katholieke Universiteit Leuven, Fakulteit Wetenschappen, DepartementWiskunde, Celestijnenlaan 200B, 3001 Heverlee, Belgium.

E-mail: leopold.verstraelen@wis.kuleuven.be 